schwerden der Verdacht einer Art "Selbstschutzreaktion" des Zweiten Senats nahe. Nicht auszuschließen ist, dass das Gericht den nach dem Wegfall der Beschwerdevoraussetzung des Beitritts von mindestens 100 Wahlberechtigten ( $\$ 48$ Abs. 1 BVerfGG a.F.) durch Art. 3 Nr. 2. a) cc) des Gesetzes zur Verbesserung des Rechtsschutzes in Wahlsachen vom 12.07.2012 (BGBl. I, S. 1501) deutlich gestiegenen Beschwerdeanfall zu kompensieren versucht. Verstärkt werden die Bedenken dadurch, dass der Senat extrem hohe Anforderungen an die $\mathrm{Zu}-$ lässigkeit von Wahlprüfungsbeschwerden stellt.

Entscheidungen im vereinfachten Verfahren nach $\$ 24$ BVerfGG bedeuten nicht, dass den Erkenntnissen keine oder eine nur geringfügige rechtliche oder praktische Relevanz zukommt. Denn auch bei einer aus prozessökonomischen Gründen erfolgenden Inanspruchnahme der Regelung hat das Gericht Gelegenheit zur Klärung wichtiger Wahlrechtsfragen und Bekräftigung oder Vertiefung bereits geäußerter Rechtsstandpunkte. In diesem Sinne unterzieht es jede Wahlprüfungsbeschwerde einer substanziellen inhaltlichen Prüfung. Entschließt es sich im Rahmen des Prüfverfahrens nach pflichtgemäßem Ermessen zu einer Veröffentlichung der Entscheidung, so hängt deren Autorität wesentlich davon ab, dass die Erkenntnisse der Öffentlichkeit in nachvollziehbarer Weise zugänglich gemacht werden. Dazu gehört, wenn in der Kurzbegründung des Verwerfungsbeschlusses darauf Bezug genommen wird, grundsätzlich auch die Veröffentlichung des die voraussichtliche Aussichtslosigkeit des Rechtsbehelfs darlegenden Schreibens des BE an den Beschwerdeführer, in vollem Umfang oder zumindest inhaltlich »in groben Zügen«. Dieses ist Teil des unter dem Gebot der Öffentlichkeit stehenden demokratischen Entscheidungsprozesses. Nur so werden die Entscheidungen verständlich. Durch Veröffentlichung auch des BE-Schreibens, in welchem Umfang und mit welchem Inhalt auch immer, leistet das Gericht einen wesentlichen Beitrag für die Offenheit des demokratischen Prozesses und die Transparenz höchstrichterlicher Erkenntnisse. Das BVerfG bleibt aufgerufen, seine Veröffentlichungspraxis in A-Limine-Verfahren nach $\$ 24$ BVerfGG zu überdenken und insoweit die aktuelle Intransparenz der Bearbeitung des größten Teils der Wahlprüfungsbeschwerden zu beseitigen. Nur vollständig veröffentlichte Entscheidungen des BVerfG in Wahlangelegenheiten sind geeignet, das Vertrauen der Bürger in die demokratischen Prozesse zu gewährleisten. In diesem Sinne sollte sich das Gericht unter das von ihm selbst, wie etwa im Wahlgeräte-Urteil vom 03.03.2009, ${ }^{61}$ betonte Gebot der Öffentlichkeit der Wahl in ihrer Gesamtheit stellen. Zudem sollte, auch wenn es keinen generellen Bearbeitungsvorrang von Wahlprüfungsverfahren gegenüber anderen Verfassungsstreitverfahren gibt, angesichts der großen staatsorganisationsrechtlichen Bedeutung der Verfahren und des ihnen immanenten "Zügigkeitsgebotes« der Klärung von Wahlfehlern doch eine gewisse "Vorfahrt" Platz greifen. Wahlprüfungsentscheidungen tragen zur Weiterentwicklung des Wahlrechts bei. Wenn das Gericht die Dauer eines Wahlprüfungsverfahrens von ca. 18 Monaten als "nicht zu beanstanden ", m.a.W. als (noch) "angemessen« beurteilt (s. unter II.3.c)), so fragt es sich, warum es diesen "Entscheidungskorridor« in mehreren die Gültigkeit der Bundestagswahl 2013 betreffenden Verfahren nicht beachtet und von der Einreichung der Beschwerde bis zur Entscheidung bis zu 37 Monate benötigt hat, ${ }^{62}$ ungeachtet der Tatsache, dass über eine Wahlprüfungsbeschwerde bis zum Ablauf der Wahlperiode des 18. BT keine Entscheidung getroffen worden ist. Um die Senatsarbeit zu entlasten und eine kürzere Verfahrensdauer zu erreichen, sollte dem schon mehrfach unterbreiteten Vorschlag nähergetreten werden, bei unzulässigen oder offensichtlich unbegründeten Wahlprüfungsbeschwerden die Einführung einer Kammerzuständigkeit (bei »Eingangszuständigkeit» des Senats) zu erwägen. ${ }^{63}$ Der Gesetzgeber wird angesichts des deutlichen Anstiegs der Wahlprüfungsbeschwerden zudem zu überdenken haben, ob die »Filterfunktion« der ersatzlos gestrichenen Beschwerdevoraussetzung des Beitritts von mindestens 100 Wahlberechtigten nicht wieder eingeführt werden sollte.

61 BVerfGE $123,39<68$ ff.>

62 Siehe z.B. die Beschl. v. 21.09.2017 - 2 BvC 29/14, 46/14, 51/14 und 64/14-.

63 Siehe insoweit bereits die Vorschläge im Bericht der vom BMJ eingesetzten Kommission "Entlastung des BVerfG" von 1997/1998, S. 19, 119 ff. und 124. Vgl. auch Schreiber, DVBl 2010, 609 (617) - Fußn. 42 und 44 und KommP Wahlen 2012, 73 (80) - mit weiteren Literaturnachweisen und einschlägigen Reformüberlegungen -, sowie Hahlen (Fußn. 1), $\$ 49$ Rdnr. 37 und 39.

\title{
598 Sitze im Bundestag statt 709? 200 Wahlkreise statt 299!
}

\author{
von Prof. Dr. Friedrich Pukelsheim, Augsburg*
}

die Ergebnisse der Personenwahl in die Verhältnisrechnung eingebettet werden können. Jedoch verheddern sich die geltenden Vorschriften in föderalen Nebensächlichkeiten, verlieren das Ziel aus den Augen und blähen dadurch den Bundestag weit mehr auf als notwendig. Eine Modellrechnung zeigt, dass die Sollgröße von 598 Sitzen dauerhaft eingehalten wird, wenn die Zahl der Wahlkreise von 299 auf 200 reduziert und die derzeitige Vorabkalkulation durch eine schlankere ersetzt wird.

\footnotetext{
* Der Autor ist emeritierter Ordinarius am Lehrstuhl für Stochastik und ihre Anwendungen im Institut für Mathematik der Universität Augsburg.
} 


\section{Einleitung}

Die Wahl zum 19. Bundestag am 24.09.2017 war nach der Wahl 2013 die zweite, in der die Verrechnung von Stimmen in Mandate mit dem Verfahren erfolgte, das 2013 in das Bundeswahlgesetz aufgenommen wurde. ${ }^{1}$ Zwar bestimmt das Gesetz weiterhin eine Sollgröße von 598 Sitzen, diese gilt aber nur "vorbehaltlich der sich aus diesem Gesetz ergebenden Abweichungen«. Der Vorbehalt kommt durch das neue Stimmenverrechnungsverfahren regelmäßig und massiv zum Tragen. Schon der 18. Bundestag konstituierte sich nicht mit 598 Sitzen, sondern mit 631. Der 19. Bundestag steigert das Übermaß auf 709 Sitze. ${ }^{2}$

In diesem Aufsatz untersuchen wir die Gründe für das übermäßige Anwachsen der Bundestagsgröße. Es schälen sich zwei Ursachen heraus. Die erste Ursache ist die zu beobachtende zunehmende Diversifizierung des Parteiensystems. Angesichts dieser Entwicklung gibt es zu viele Direktmandate, um sie reibungsfrei in die Verhältnisrechnung einbetten zu können. Diese Ursache hat strukturellen Charakter und ist weitgehend unabhängig von den Einzelheiten der Stimmenverrechnung. Dagegen liegt die zweite Ursache in der überaus schwerfälligen und wenig sachgerechten Vorabkalkulation der Bundestagsgröße, die das BWG2013 vorschreibt. Dieses mehr technische Defizit kann durch eine schlankere Bestimmung der Bundestagsgröße leicht behoben werden.

Der Aufsatz ist folgendermaßen gegliedert. Zunächst wird nachvollzogen, wie das BWG2013 die Stimmenergebnisse bei der Wahl 2017 auswertet und schließlich einen Bundestag mit 709 Sitzen etabliert (II). Das vorher gültige BWG2008 wäre wegen zu vieler Überhangmandate in eine verfassungswidrige Sackgasse gelaufen (III). Aktuelle Übergröße und vormalige Überhangmandate verweisen auf die Unausgewogenheit der Verbindung von Personenwahl und Verhältniswahl (IV). Eine ausgewogene Verbindung kommt zustande, wenn bezogen auf die Sollgröße die Zahl der Direktmandate von der Hälfte (299) auf ein Drittel (200) reduziert wird (V). Trotz Reduzierung würde das BWG2013 erhebliche Schwächen aufweisen (VI). Diese können durch eine Verschlankung der Vorschriften behoben werden (VII). Das verschlankte System und das BWG2013 sind hinsichtlich des föderalen Proporzverlustes gleichwertig (VIII). Die Reduzierung der Zahl der Wahlkreise dürfte wohl eine politische Herausforderung darstellen und sollte einer ruhigen Vorprüfung unterzogen werden (IX).

\section{Die 709 Sitze im 19. Bundestag}

Am leichtesten erklärt sich die im BWG2013 normierte Auswertung der Wahl vom 24.09.2017, ${ }^{3}$ wenn wir das Pferd von hinten aufzäumen und erst einmal hinnehmen, dass der 19. Bundestag 709 Sitze hat.

Die 709 Gesamtsitze werden mit dem Divisorverfahren mit Standardrundung den zu berücksichtigenden Parteien im Verhältnis ihrer bundesweiten Zweitstimmenergebnisse zugeteilt; das Verfahren wird in Deutschland auch nach dem Franzosen André Sainte-Laguë (1852-1950) und dem Deutschen Hans Schepers $\left({ }^{*} 1928\right)$ benannt. Das Ergebnis steht in Spalte J von Schaubild 2: Aufje 62.300 Zweitstimmen entfällt rund ein Sitz. Der Bundesdivisor 62.300 ist so berechnet, dass genau 709 Sitze vergeben werden, nicht mehr und auch nicht weniger.

Für die CSU ist das Verfahren hier zu Ende, sie bekommt 46 Sitze zugeteilt. Da sie auch 46 Direktmandate gewonnen hat, werden alle CSU-Sitze durch Wahlkreissieger eingenommen. Die CSU-Landesliste hat das Nachsehen und geht leer aus.

Für jede der anderen Parteien müssen die Sitze aus der Oberzuteilung weiter gereicht werden an die Landeslisten der Partei. Dies geschieht mit der direktmandatsbedingten Variante, die mit dem Divisorverfahren mit Standardrundung einhergeht. Die sechs Unterzuteilungen an die Parteien CDU, SPD, AfD, FDP, LINKE und GRÜNE stehen im unteren Teil von Schaubild 2. Zum Beispiel ergibt sich für die CDU: Aufje 76.000 Zweitstimmen einer CDU-Landesliste entfällt rund ein Sitz, außer wenn die CDU-Direktmandate in diesem Land mehr Sitze erfordern. In Schaubild 2 sind diejenigen Quotienten aus Zweitstimmen und Parteidivisor, die wegen überschießender Direktmandate keine Wirkung entfalten, durchgestrichen. Der Parteidivisor ist so berechnet, dass die Sitzzahlen, die sich mit der direktmandatsbedingten Variante ergeben, in der Summe die Parteisitze aus der Oberzuteilung genau ausschöpfen. ${ }^{4}$ Auf diese Weise bekommt jede Landesliste hinreichend viele Sitze zugeteilt, um alle ihre Wahlkreissieger mit einem Mandat zu versehen. Darüber hinaus werden die Mandate aus der Landesliste besetzt wie früher auch.

Die direktmandatsbedingte Zuteilungsvariante würde scheitern, wenn die Sollgröße von 598 Sitzen verpflichtend wäre. Dann entfielen auf die CDU nur 168 Verhältnissitze ${ }^{5}$ und es wäre unmöglich, die 185 CDU-Direktmandate in die Unterzuteilung einzubetten. Bei der CSU ist dieselbe Unvereinbarkeit zu beobachten; für 46 Direktmandate ständen nur 39 Verhältnissitze bereit. Um nicht an Unterdeckung zu scheitern, wird in einer Vorabkalkulation die Bundestagsgröße angehoben, damit für jede Partei die direktmandatsbedingte Unterzuteilung erfolgreich vollzogen werden kann. Im BWG2013 landet die Vorabkalkulation bei einer Bundestagsgröße von 709 Sitzen.

1 Im Folgenden zitiert als BWG2013; siehe Zweiundzwanzigstes Gesetz zu Änderung des Bundeswahlgesetzes vom 03.05.2013 (BGBl. I, S. 1082) Der Weg zur Reform 2013 war reich an Hindernissen und nicht frei von Irrungen und Wirrungen, siehe J. Behnke/F. Grotz/C. Hartmann, Wahlen und Wahlsysteme, Berlin 2017 [169-178]. Zur Genesis der Novellierungen des Bundeswahlgesetztes siehe W. Schreiber, BWahlG - Bundeswahlgesetz Kommentar - 10. Aufl., Köln 2017 [75-77].

2 Schlimmer noch: Bei Anwendung des BWG2013 wäre in allen früheren Wahlen die Sollgröße des Bundestags zu Makulatur geworden. Siehe F. Pukelsheim/ M. Rossi, Imperfektes Wahlrecht, ZG 28 (2013) 209-226 [222].

3 Die Daten entstammen den Informationen des Bundeswahlleiters, Bundestagswahl 2017, Heft 3: Endgültige Ergebnisse nach Wahlkreisen [379-427]. S.a. B. Stemmer, Endgültiges Ergebnis der Wahl zum 19. Deutschen Bundestag am 24.09.2017, Statistisches Bundesamt, Wirtschaft und Statistik 6/2017, 74-94. Die Auswertung gem. \$6 BWG2013 kommentiert Schreiber, Fußn. 1 [209-263].

4 Die Sitzzahl einer Landesliste ist also das Maximum der Zahl ihrer Direktmandate in diesem Land und des gerundeten Quotienten aus Zweitstimmen und Parteidivisor. Die Maximumsbildung unterscheidet die direktmandatsbedingte Variante vom originären Divisorverfahren mit Standardrundung; die beiden Zuteilungsverfahren sind nicht identisch. Auf die direktmandatsbedingte Variante darf deshalb nicht mit dem Namenszusatz "Sainte-Laguë/Schepers" verwiesen werden. Zur Unterscheidung wurde für die direktmandatsbedingte Variante der Namenszusatz »Augsburger Zuteilungsverfahren" propagiert, siehe Pukelsheim, DVBl 123 (2008) 889-897 [889]; Pukelsheim/Rossi, Fußn. 2 [214]. Im vorliegenden Aufsatz verzichten wir auf Namenszusätze und sprechen schlicht vom "Divisorverfahren mit Standardrundung « und von der »direktmandatsbedingten Variante«. Es kommt durchaus vor, dass beide Verfahren im Ergebnis übereinstimmen. Dies passiert immer dann, wenn alle Direktmandatszahlen kleiner oder gleich den gerundeten Quotienten sind (AfD, FDP, LINKE, GRÜNE).

5 Spalte E in Schaubild 2. 


\begin{tabular}{|c|c|c|c|c|c|}
\hline $\mathrm{A}$ & $\mathrm{B}$ & $\mathrm{C}$ & $\mathrm{D}$ & $\mathrm{E}$ & $\mathrm{F}$ \\
\hline \multirow{2}{*}{ Landeskürzel } & \multirow{2}{*}{ Land } & \multirow{2}{*}{$\begin{array}{l}\text { Deutsche Bevölkerung } \\
\text { am 30.06.2016 }\end{array}$} & \multicolumn{3}{|c|}{ Vorabkalkulation Schritte 1 und 2} \\
\hline & & & ungerundet & Sitzkontingent & Landesdivisor \\
\hline $\mathrm{SH}$ & Schleswig-Holstein & 2.673 .803 & 21,8 & 22 & 80.000 \\
\hline MV & Mecklenburg-Vorpommern & 1.548 .400 & 12,6 & 13 & 68.600 \\
\hline $\mathrm{HH}$ & Hamburg & 1.525 .090 & 12,4 & 12 & 78.000 \\
\hline $\mathrm{NI}$ & Niedersachsen & 7.278 .789 & 59,4 & 59 & 76.000 \\
\hline $\mathrm{HB}$ & Bremen & 568.510 & 4,6 & 5 & 64.000 \\
\hline $\mathrm{BB}$ & Brandenburg & 2.391 .746 & 19,504 & 20 & 71.000 \\
\hline ST & Sachsen-Anhalt & 2.145 .671 & 17,497 & 17 & 69.000 \\
\hline $\mathrm{BE}$ & Berlin & 2.975 .745 & 24,3 & 24 & 70.000 \\
\hline NW & Nordrhein-Westfalen & 15.707 .569 & 128,1 & 128 & 74.000 \\
\hline SN & Sachsen & 3.914 .671 & 31,9 & 32 & 74.000 \\
\hline $\mathrm{HE}$ & Hessen & 5.281 .198 & 43,1 & 43 & 74.000 \\
\hline $\mathrm{TH}$ & Thüringen & 2.077 .901 & 16,9 & 17 & 68.000 \\
\hline $\mathrm{RP}$ & Rheinland-Pfalz & 3.661 .245 & 29,9 & 30 & 75.000 \\
\hline BY & Bayern & 11.362 .245 & 92,7 & 93 & 73.000 \\
\hline BW & Baden-Württemberg & 9.365 .001 & 76,4 & 76 & 76.000 \\
\hline SL & Saarland & 899.748 & 7,3 & 7 & 80.000 \\
\hline \multicolumn{2}{|c|}{ Summe (Verteilungsschlüssel) } & 73.377 .332 & $(122.630)$ & 598 & - \\
\hline
\end{tabular}

\begin{tabular}{|c|c|c|c|c|c|c|c|c|c|c|c|c|c|c|}
\hline $\mathrm{K}$ & $\mathrm{L}$ & $\mathrm{M}$ & $\mathrm{N}$ & $\mathrm{O}$ & $\mathrm{K}$ & $\mathrm{L}$ & $\mathrm{M}$ & $\mathrm{N}$ & $\mathrm{O}$ & $\mathrm{K}$ & $\mathrm{L}$ & $\mathrm{M}$ & $\mathrm{N}$ & $\mathrm{O}$ \\
\hline Landesliste & Dir. & Zweitst. & Quotient & Fikt. & Landesliste & Dir. & Zweitst. & Quotient & Fikt. & Landesliste & Dir. & Zweitst. & Quotient & Fikt. \\
\hline & & & & & & & & & & & & & & \\
\hline \multicolumn{5}{|c|}{ CDU: Länderweise Fiktivsitze } & \multicolumn{5}{|c|}{ SPD: Länderweise Fiktivsitze } & \multicolumn{5}{|c|}{ AfD: Länderweise Fiktivsitze } \\
\hline $\mathrm{SH}$ & 10 & 583.135 & 7,3 & 10 & $\mathrm{SH}$ & 1 & 399.505 & 5,0 & 5 & $\mathrm{SH}$ & 0 & 140.362 & 1,8 & 2 \\
\hline MV & 6 & 307.263 & 4,48 & 6 & MV & 0 & 139.689 & 2,0 & 2 & MV & 0 & 172.409 & 2,51 & 3 \\
\hline $\mathrm{HH}$ & 1 & 266.312 & 3,4 & 3 & $\mathrm{HH}$ & 5 & 229.862 & 2,9 & 5 & $\mathrm{HH}$ & 0 & 76.511 & 1,0 & 1 \\
\hline $\mathrm{NI}$ & 16 & 1.623 .481 & 21,4 & 21 & $\mathrm{NI}$ & 14 & 1.275 .172 & 16,8 & \begin{tabular}{l|l}
17 \\
\end{tabular} & $\mathrm{NI}$ & 0 & 422.362 & 5,6 & 6 \\
\hline $\mathrm{HB}$ & 0 & 83.409 & 1,3 & 1 & $\mathrm{HB}$ & 2 & 88.944 & 7,4 & 2 & $\mathrm{HB}$ & 0 & 33.244 & 0,52 & 1 \\
\hline $\mathrm{BB}$ & 9 & 397.839 & 5,6 & 9 & $\mathrm{BB}$ & 1 & 261.822 & 3,7 & 4 & $\mathrm{BB}$ & 0 & 301.103 & 4,2 & \\
\hline ST & 9 & 377.411 & 5,47 & 9 & ST & 0 & 188.980 & 2,7 & 3 & ST & 0 & 244.401 & 3,54 & 4 \\
\hline $\mathrm{BE}$ & 4 & 424.321 & 6,1 & 6 & $\mathrm{BE}$ & 3 & 334.253 & 4,8 & 5 & $\mathrm{BE}$ & 0 & 225.170 & 3,2 & 3 \\
\hline $\mathrm{NW}$ & 38 & 3.214 .013 & 43,4 & 43 & $\mathrm{NW}$ & 26 & 2.557 .876 & 34,6 & 35 & NW & 0 & 928.425 & 12,55 & 13 \\
\hline SN & 12 & 665.751 & $9, \theta$ & 12 & SN & 0 & 261.105 & 3,53 & 4 & SN & 3 & 669.940 & 9,1 & 9 \\
\hline $\mathrm{HE}$ & 17 & 1.033 .200 & $\pm 4,0$ & 17 & $\mathrm{HE}$ & 5 & 788.427 & 10,7 & 11 & $\mathrm{HE}$ & 0 & 398.712 & 5,4 & 5 \\
\hline $\mathrm{TH}$ & 8 & 372.258 & 5,47 & 8 & $\mathrm{TH}$ & 0 & 171.032 & 2,52 & 3 & $\mathrm{TH}$ & 0 & 294.069 & 4,3 & 4 \\
\hline $\mathrm{RP}$ & 14 & 848.003 & $\pm 1,3$ & 14 & $\mathrm{RP}$ & 1 & 570.518 & 7,6 & 8 & $\mathrm{RP}$ & 0 & 265.688 & 3,54 & 4 \\
\hline BY & - & - & - & - & BY & 0 & 1.130 .931 & 15,49 & 15 & BY & 0 & 916.300 & 12,6 & 13 \\
\hline BW & 38 & 2.061 .687 & 27,1 & 38 & BW & 0 & 982.370 & 12,9 & 13 & BW & 0 & 730.499 & 9,6 & 10 \\
\hline SL & 3 & 189.573 & 2,4 & 3 & SL & 1 & 158.895 & 2,0 & 2 & SL & 0 & 58.920 & 0,7 & 1 \\
\hline \multicolumn{4}{|c|}{ Mindestsitzzahl (= Sa. Fiktivsitze) } & 200 & \multicolumn{4}{|c|}{ Mindestsitzzahl (= Sa. Fiktivsitze) } & 134 & \multicolumn{4}{|c|}{ Mindestsitzzahl (= Sa. Fiktivsitze) } & 83 \\
\hline \multicolumn{5}{|c|}{ FDP: Länderweise Fiktivsitze } & \multicolumn{5}{|c|}{ LINKE: Länderweise Fiktivsitze } & \multicolumn{5}{|c|}{ GRÜNE: Länderweise Fiktivsitze } \\
\hline SH & 0 & 216.844 & 2,7 & 3 & $\mathrm{SH}$ & 0 & 124.678 & 1,6 & $2 \mid$ & $\mathrm{SH}$ & 0 & 205.471 & 2,6 & 3 \\
\hline MV & 0 & 57.895 & 0,8 & 1 & MV & 0 & 165.368 & 2,4 & $2 \mid$ & MV & 0 & 39.514 & 0,6 & 1 \\
\hline $\mathrm{HH}$ & 0 & 105.610 & 1,4 & 1 & $\mathrm{HH}$ & 0 & 119.076 & 1,53 & 2 & $\mathrm{HH}$ & 0 & 136.371 & 1,7 & 2 \\
\hline $\mathrm{NI}$ & 0 & 431.405 & 5,7 & 6 & $\mathrm{NI}$ & 0 & 322.979 & 4,2 & 4 & NI & 0 & 404.825 & 5,3 & 5 \\
\hline $\mathrm{HB}$ & 0 & 31.056 & 0,49 & 0 & $\mathrm{HB}$ & 0 & 44.629 & 0,7 & 1 & $\mathrm{HB}$ & 0 & 36.733 & 0,6 & 1 \\
\hline BB & 0 & 105.485 & 1,49 & 1 & BB & 0 & 255.721 & 3,6 & 4 & BB & 0 & 74.971 & 1,1 & 1 \\
\hline ST & 0 & 96.555 & 1,4 & 1 & ST & 0 & 220.858 & 3,2 & 3 & ST & 0 & 46.243 & 0,7 & 1 \\
\hline $\mathrm{BE}$ & 0 & 167.046 & 2,4 & 2 & $\mathrm{BE}$ & 4 & 351.170 & 5,0 & 5 & $\mathrm{BE}$ & 1 & 234.947 & 3,4 & 3 \\
\hline $\mathrm{NW}$ & 0 & 1.293 .052 & 17,47 & 17 & $\mathrm{NW}$ & 0 & 736.904 & 10,0 & 10 & $\mathrm{NW}$ & 0 & 744.970 & 10,1 & 10 \\
\hline $\mathrm{SN}$ & 0 & 203.662 & 2,8 & 3 & $\mathrm{SN}$ & 1 & 398.627 & 5,4 & 5 & SN & 0 & 113.608 & 1,54 & 2 \\
\hline $\mathrm{HE}$ & 0 & 386.742 & 5,2 & 5 & $\mathrm{HE}$ & 0 & 271.158 & 3,7 & 4 & $\mathrm{HE}$ & 0 & 323.736 & 4,4 & 4 \\
\hline $\mathrm{TH}$ & 0 & 101.129 & 1,49 & 1 & TH & 0 & 218.212 & 3,2 & 3 & $\mathrm{TH}$ & 0 & 53.340 & 0,8 & 1 \\
\hline $\mathrm{RP}$ & 0 & 245.235 & 3,3 & 3 & $\mathrm{RP}$ & 0 & 160.912 & 2,1 & 2 & $\mathrm{RP}$ & 0 & 179.233 & 2,4 & 2 \\
\hline BY & 0 & 751.248 & 10,3 & 10 & BY & 0 & 450.803 & 6,2 & 6 & BY & 0 & 722.116 & 9,9 & 10 \\
\hline $\mathrm{BW}$ & 0 & 762.008 & 10,0 & 10 & $\mathrm{BW}$ & 0 & 380.727 & 5,0 & 5 & $\mathrm{BW}$ & 0 & 807.205 & 10,6 & 11 \\
\hline SL & 0 & 44.477 & 0,6 & 1 & SL & 0 & 75.448 & 0,9 & 1 & SL & 0 & 35.117 & 0,4 & 0 \\
\hline \multicolumn{4}{|c|}{ Mindestsitzzahl (= Sa. Fiktivsitze) } & 65 & \multicolumn{4}{|c|}{ Mindestsitzzahl (= Sa. Fiktivsitze) } & 59 & \multicolumn{4}{|c|}{ Mindestsitzzahl (= Sa. Fiktivsitze) } & 57 \\
\hline
\end{tabular}

\section{Schaubild 1: Sitzkontingente der Länder nach Bevölkerungszahl und Mindestsitzzahlen der Parteien}

Spalten D und E: Sitzkontingente der Länder bei 598 Nominalsitzen (Schritt 1 der Vorabkalkulation). Spalte N: Quotient aus Zweitstimmen und Landesdivisor; der Landesdivisor (Spalte F) ist so bestimmt, dass im Land die gerundeten Quotienten in der Summe das Sitzkontingent genau ausschöpfen. Spalte O: Die Fiktivsitze ergeben sich entweder als gerundeter Quotient oder, falls größer, als Zahl der Direktmandate in Spalte L. Die Summe (Sa.) der Fiktivsitze einer Partei gilt als Mindestsitzzahl dieser Partei (Schritt 2 der Vorabkalkulation). Die CSU kommt mit Quotient 2.869.688/73.000 = 39,3 und 46 Direktmandaten auf Mindestsitzzahl 46. 


\begin{tabular}{|c|c|c|c|c|c|c|c|c|}
\hline A & B & $\mathrm{C}$ & $\mathrm{D}$ & $\mathrm{E}$ & $\mathrm{F}$ & G & $\mathrm{H}$ & $\mathrm{J}$ \\
\hline 19. WP 2017 & \multirow{2}{*}{$\begin{array}{c}\text { Mindest- } \\
\text { sitzzahl }\end{array}$} & \multirow{2}{*}{$\begin{array}{c}\text { Zweit- } \\
\text { stimmen }\end{array}$} & \multicolumn{2}{|c|}{ Anfangsgröße 598} & \multicolumn{2}{|c|}{ Zwischengröße 708} & \multicolumn{2}{|c|}{ Endgröße 709} \\
\hline Partei & & & Quotient & Sitze & Quotient & Sitze & Quotient & Sitze \\
\hline $\mathrm{CDU}$ & 200 & 12.447 .656 & 168,4 & $168^{\bullet}$ & 199,2 & $199 \bullet$ & 199,8 & 200 \\
\hline SPD & 134 & 9.539 .381 & 129,1 & $129 \bullet$ & 152,6 & 153 & 153,1 & 153 \\
\hline AfD & 83 & 5.878 .115 & 79,54 & $80 \bullet$ & 94,0 & 94 & 94,4 & 94 \\
\hline FDP & 65 & 4.999 .449 & 67,7 & 68 & 80,0 & 80 & 80,2 & 80 \\
\hline LINKE & 59 & 4.297 .270 & 58,1 & $58^{\bullet}$ & 68,8 & 69 & 69,0 & 69 \\
\hline GRÜNE & 57 & 4.158 .400 & 56,3 & $56 \bullet$ & 66,53 & 67 & 66,7 & 67 \\
\hline $\mathrm{CSU}$ & 46 & 2.869 .688 & 38,8 & $39 \bullet$ & 45,9 & 46 & 46,1 & 46 \\
\hline Summe (Bundesdivisor) & 644 & 44.189 .959 & $(73.900)$ & 598 & $(62.500)$ & 708 & $(62.300)$ & 709 \\
\hline
\end{tabular}

\begin{tabular}{|c|c|c|c|c|c|c|c|c|c|c|c|c|c|c|}
\hline $\mathrm{K}$ & $\mathrm{L}$ & $M$ & $\mathrm{~N}$ & $\mathrm{O}$ & $\mathrm{K}$ & $\mathrm{L}$ & $\mathrm{M}$ & $\mathrm{N}$ & $\mathrm{O}$ & $\mathrm{K}$ & $\mathrm{L}$ & $M$ & $\mathrm{~N}$ & $\mathrm{O}$ \\
\hline $\begin{array}{l}\text { Landes- } \\
\text { liste }\end{array}$ & Dir. & Zweitst. & Quotient & Sitze & $\begin{array}{l}\text { Landes- } \\
\text { liste }\end{array}$ & Dir. & Zweitst. & Quotient & Sitze & $\begin{array}{l}\text { Landes- } \\
\text { liste }\end{array}$ & Dir. & Zweitst. & Quotient & $\begin{array}{l}\text { Sit- } \\
\text { ze }\end{array}$ \\
\hline \multicolumn{5}{|c|}{ Unterzuteilung für die CDU } & \multicolumn{5}{|c|}{ Unterzuteilung für die SPD } & \multicolumn{5}{|c|}{ Unterzuteilung für die AfD } \\
\hline $\mathrm{SH}$ & 10 & 583.135 & 7,7 & 10 & $\mathrm{SH}$ & 1 & 399.505 & 6,3 & 6 & $\mathrm{SH}$ & 0 & 140.362 & 2,2 & 2 \\
\hline MV & 6 & 307.263 & $4, \theta$ & 6 & MV & 0 & 139.689 & 2,2 & 2 & MV & 0 & 172.409 & 2,7 & 3 \\
\hline $\mathrm{HH}$ & 1 & 266.312 & 3,504 & 4 & $\mathrm{HH}$ & 5 & 229.862 & 3,6 & 5 & $\mathrm{HH}$ & 0 & 76.511 & 1,2 & 1 \\
\hline NI & 16 & 1.623 .481 & 21,4 & 21 & NI & 14 & 1.275 .172 & 20,2 & 20 & NI & 0 & 422.362 & 6,6 & 7 \\
\hline $\mathrm{HB}$ & 0 & 83.409 & 1,1 & 1 & $\mathrm{HB}$ & 2 & 88.944 & 1,4 & 2 & $\mathrm{HB}$ & 0 & 33.244 & 0,52 & 1 \\
\hline BB & 9 & 397.839 & 5,2 & 9 & $\mathrm{BB}$ & 1 & 261.822 & 4,1 & 4 & $\mathrm{BB}$ & 0 & 301.103 & 4,7 & 5 \\
\hline ST & 9 & 377.411 & $5, \theta$ & 9 & ST & 0 & 188.980 & 3,0 & 3 & ST & 0 & 244.401 & 3,8 & 4 \\
\hline $\mathrm{BE}$ & 4 & 424.321 & 5,6 & 6 & $\mathrm{BE}$ & 3 & 334.253 & 5,3 & 5 & $\mathrm{BE}$ & 0 & 225.170 & 3,53 & 4 \\
\hline $\mathrm{NW}$ & 38 & 3.214 .013 & 42,3 & 42 & NW & 26 & 2.557 .876 & 40,54 & 41 & NW & 0 & 928.425 & 14,6 & 15 \\
\hline SN & 12 & 665.751 & 8,8 & 12 & SN & 0 & 261.105 & 4,1 & 4 & SN & 3 & 669.940 & 10,52 & 11 \\
\hline $\mathrm{HE}$ & 17 & 1.033 .200 & 13,6 & 17 & $\mathrm{HE}$ & 5 & 788.427 & 12,49 & 12 & $\mathrm{HE}$ & 0 & 398.712 & 6,3 & 6 \\
\hline $\mathrm{TH}$ & 8 & 372.258 & 4,9 & 8 & $\mathrm{TH}$ & 0 & 171.032 & 2,7 & 3 & TH & 0 & 294.069 & 4,6 & 5 \\
\hline $\mathrm{RP}$ & 14 & 848.003 & 11,2 & 14 & $\mathrm{RP}$ & 1 & 570.518 & 9,0 & 9 & $\mathrm{RP}$ & 0 & 265.688 & 4,2 & 4 \\
\hline BY & - & - & - & - & BY & 0 & 1.130 .931 & 17,9 & 18 & $\mathrm{BY}$ & 0 & 916.300 & 14,4 & 14 \\
\hline $\mathrm{BW}$ & 38 & 2.061 .687 & 27,1 & 38 & BW & 0 & 982.370 & 15,6 & 16 & $\mathrm{BW}$ & 0 & 730.499 & 11,47 & 11 \\
\hline SL & 3 & 189.573 & 2,49 & 3 & SL & 1 & 158.895 & 2,52 & 3 & SL & 0 & 58.920 & 0,9 & 1 \\
\hline Sa. (Div.) & 185 & 12.447 .656 & $(76.000)$ & 200 & Sa. (Div.) & 59 & 9.539 .381 & $(63.100)$ & 153 & Sa. (Div.) & 3 & 5.878 .115 & $(63.700)$ & 94 \\
\hline \multicolumn{5}{|c|}{ Unterzuteilung für die FDP } & \multicolumn{5}{|c|}{ Unterzuteilung für die LINKE } & \multicolumn{5}{|c|}{ Unterzuteilung für die GRÜNEN } \\
\hline $\mathrm{SH}$ & 0 & 216.844 & 3,4 & 3 & $\mathrm{SH}$ & 0 & 124.678 & 2,0 & 2 & $\mathrm{SH}$ & 0 & 205.471 & 3,2 & 3 \\
\hline MV & 0 & 57.895 & 0,9 & 1 & MV & 0 & 165.368 & 2,6 & 3 & MV & 0 & 39.514 & 0,6 & 1 \\
\hline $\mathrm{HH}$ & 0 & 105.610 & 1,7 & 2 & $\mathrm{HH}$ & 0 & 119.076 & 1,9 & 2 & $\mathrm{HH}$ & 0 & 136.371 & 2,1 & 2 \\
\hline $\mathrm{NI}$ & 0 & 431.405 & 6,7 & 7 & $\mathrm{NI}$ & 0 & 322.979 & 5,1 & 5 & $\mathrm{NI}$ & 0 & 404.825 & 6,3 & 6 \\
\hline $\mathrm{HB}$ & 0 & 31.056 & 0,49 & 0 & $\mathrm{HB}$ & 0 & 44.629 & 0,7 & 1 & $\mathrm{HB}$ & 0 & 36.733 & 0,6 & 1 \\
\hline BB & 0 & 105.485 & 1,6 & 2 & $\mathrm{BB}$ & 0 & 255.721 & 4,1 & 4 & $\mathrm{BB}$ & 0 & 74.971 & 1,2 & 1 \\
\hline ST & 0 & 96.555 & 1,51 & 2 & ST & 0 & 220.858 & 3,51 & 4 & ST & 0 & 46.243 & 0,7 & 1 \\
\hline $\mathrm{BE}$ & 0 & 167.046 & 2,6 & 3 & $\mathrm{BE}$ & 4 & 351.170 & 5,6 & 6 & $\mathrm{BE}$ & 1 & 234.947 & 3,7 & 4 \\
\hline $\mathrm{NW}$ & 0 & 1.293 .052 & 20,2 & 20 & NW & 0 & 736.904 & 11,7 & 12 & $\mathrm{NW}$ & 0 & 744.970 & 11,6 & 12 \\
\hline SN & 0 & 203.662 & 3,2 & 3 & SN & 1 & 398.627 & 6,3 & 6 & SN & 0 & 113.608 & 1,8 & 2 \\
\hline $\mathrm{HE}$ & 0 & 386.742 & 6,0 & 6 & $\mathrm{HE}$ & 0 & 271.158 & 4,3 & 4 & $\mathrm{HE}$ & 0 & 323.736 & 5,1 & 5 \\
\hline $\mathrm{TH}$ & 0 & 101.129 & 1,6 & 2 & $\mathrm{TH}$ & 0 & 218.212 & 3,46 & 3 & $\mathrm{TH}$ & 0 & 53.340 & 0,8 & 1 \\
\hline $\mathrm{RP}$ & 0 & 245.235 & 3,8 & 4 & $\mathrm{RP}$ & 0 & 160.912 & 2,6 & 3 & $\mathrm{RP}$ & 0 & 179.233 & 2,8 & 3 \\
\hline BY & 0 & 751.248 & 11,7 & 12 & BY & 0 & 450.803 & 7,2 & 7 & BY & 0 & 722.116 & 11,3 & 11 \\
\hline BW & 0 & 762.008 & 11,9 & 12 & $\mathrm{BW}$ & 0 & 380.727 & 6,0 & 6 & $\mathrm{BW}$ & 0 & 807.205 & 12,6 & 13 \\
\hline SL & 0 & 44.477 & 0,7 & 1 & SL & 0 & 75.448 & 1,2 & 1 & SL & 0 & 35.117 & 0,55 & 1 \\
\hline Sa. (Div.) & 0 & 4.999 .449 & $(64.000)$ & 80 & Sa. (Div.) & 5 & 4.297 .270 & \begin{tabular}{|c|}
$(63.000)$ \\
\end{tabular} & 69 & Sa. (Div.) & 1 & 4.158 .400 & $(64.000)$ & 67 \\
\hline
\end{tabular}

Schaubild 2: Bestimmung der Bundestagsgröße, Oberzuteilung auf Bundesebene und Unterzuteilungen für die Parteien

Spalten D bis J: Bei 598 Gesamtsitzen verfehlen sechs Parteien ihre Mindestsitzzahl (•), bei 708 Gesamtsitzen noch eine, bei 709 keine mehr (Schritt 3 der Vorabkalkulation). Spalte J enthält die Oberzuteilung der 709 Sitze an die Parteien: Aufje 62.300 Zweitstimmen entfällt rund ein Sitz. Spalte O: Die Unterzuteilung an die Landeslisten einer Partei ergibt sich entweder als gerundeter Quotient (Spalte N) aus Zweitstimmen und Parteidivisor (Div.) oder, falls größer, als Zahl der Direktmandate (Spalte L); der Parteidivisor ist so bestimmt, dass die Sitze für die Landeslisten in der Summe die Parteisitze aus der Oberzuteilung genau ausschöpfen. 
Die Vorabkalkulation der Bundestagsgröße gerät im BWG2013 germanisch-erdenschwer. Schaubild 1 zwingt die ausufernden Rechnungen auf eine Druckseite. Erstens werden die 598 Sollsitze gemäß Bevölkerungszahl auf die Länder verteilt. Die zweite Stufe ist ein Mammutprogramm, das jedes Land einzeln abhandelt. Das Sitzkontingent des Landes wird gemäß Zweitstimmen den dortigen Landeslisten zugeteilt. Zum Beispiel erhält man in Schleswig-Holstein mit Landesdivisor 80.000 für CDU, SPD, AfD, FDP, LINKE und GRÜNE aus den Quotienten 7,3, 5,0, 1,8, 2,7, 1,6 und 2,6 die Interimssitzzahlen 7, 5, 2, 3, 2 und 3. Der Landesdivisor 80.000 stellt sicher, dass das schleswig-holsteinische Kontingent von 22 Sitzen voll ausgeschöpft wird. Nun wird für jede Partei die Interimssitzzahl mit der Zahl ihrer Direktmandate verglichen. Das bessere der beiden Ergebnisse wird als Zahl der "Fiktivsitze« vorgemerkt. In Schleswig-Holstein hat die CDU 10 Wahlkreise gewonnen und springt somit auf 10 Fiktivsitze; für die anderen Parteien sind die Fiktivsitze gleich den Interimssitzen. ${ }^{6}$ Auf diese Weise kommen für jede Partei in jedem Land Fiktivsitze zustande. Die Summe der Fiktivsitze einer Partei wird als Mindestsitzzahl dieser Partei vorgemerkt. Die Mindestsitzzahlen für CDU, SPD, AfD, FDP, LINKE und GRÜNE sind 200, 134, 83, 65, 59 und 57.

Drittens und letztens wird mit dem Divisorverfahren mit Standardrundung geprüft, ob bei der Zuteilung von 598 Sollsitzen jede Partei ihre Mindestsitzzahl erreicht. Dies ist nicht der Fall. Also wird die Bundestagsgröße erhöht. Erst ab einer Gesamtzahl von 709 Sitzen werden alle Mindestsitzzahlen erreicht. ${ }^{7}$ Somit wird die Bundestagsgröße auf 709 Sitze festgelegt. Die Vorabkalkulation ist erledigt.

\section{Verfassungswidrigkeit des BWG2008}

Mit 709 Sitzen ist der 19. Bundestag so groß wie nie zuvor. Die Übergröße des Bundestages mag zunächst irritieren, aber wir bekommen etwas Wichtiges dafür: Verfassungskonformität.

Denn weil das Divisorverfahren mit Standardrundung auf alle 709 Sitze angewendet wird, ist der Erfolgswertgleichheit der Wählerstimmen in einer bestmöglichen Weise bundesweit Genüge getan. ${ }^{8}$ Erfolgswertgleichheit gilt auch bei den Unterzuteilungen an die Landeslisten für AfD, FDP, LINKE und GRÜNE, weil für diese Parteien das Zuteilungsergebnis mit der direktmandatsbedingten Variante identisch ist mit dem, das mit dem Divisorverfahren mit Standardrundung zustande kommt. Nur bei den Unterzuteilungen für CDU und SPD treten föderale Unproportionalitäten auf, um allen Direktmandatsgewinnen genügen zu können. ${ }^{?}$

Dagegen hätte die früher übliche Rechnung mit dem BWG2008 ${ }^{10}$ zu vierundvierzig Überhangmandate geführt: vierunddreißig für die CDU, drei für die SPD und sieben für die CSU.

Im gespaltenen Urteil des Bundesverfassungsgerichts vom 10.04.1997 warfen die vier das Urteil tragenden Richter eine Fünfprozent-Grenze in die Debatte, jenseits der eine verfassungsrechtliche Beanstandung von Überhangmandaten angezeigt sein könnte. ${ }^{11}$ Vierundvierzig Überhangmandate machen bei einer Sollgröße von 598 Sitzen 7,4\% aus und hätten die Fünfprozent-Grenze weit hinter sich gelassen. Sie wären also auch für Überhang-Aficionados (vermutlich) inakzeptabel. Außerdem meinten die vier Richter, es sei nicht abzusehen, dass die Zahl der Überhangmandate bei künftigen Wahlen weiter erheblich ansteigen werde. Die politische Wirklichkeit belehrt uns eines Besseren. ${ }^{12}$

Im Urteil vom 25.07.2012 hat das Bundesverfassungsgericht den Gürtel enger geschnallt und als verfassungsrechtliche Obergrenze für Überhangmandate etwa eine halbe Fraktionsstärke definiert. ${ }^{13}$ Vierundvierzig Überhangmandate sind ein Dreifaches davon, anderthalb Fraktionsstärken. Mit diesem Maßstab hätte das BWG2008 bei der Wahl 2017 zu einem verfassungswidrigen Bundestag geführt. Eine Rückkehr zum BWG2008 ist ausgeschlossen.

\section{Problem: Unausgewogene Verbindung von Personenwahl und Verhältniswahl}

Aktuelle Übergröße und vormalige Überhangmandate verweisen auf ein strukturelles Problem, dass wenig bis nichts mit den Einzelheiten des Verfahrens zu tun hat, wie Stimmen in Mandate verrechnet werden. Das Problem ist, dass unter den heutigen politischen Verhältnissen Personenwahl und Verhältniswahl zu unausgewogen nebeneinanderstehen, um widerspruchsfrei miteinander verbunden werden zu können.

Angesichts der Diversifizierung des Parteiensystems erhält die stärkste Gruppierung nur noch etwa ein Drittel der zuteilungsberechtigten Zweitstimmen. Aber sie bleibt stärker als die Mitbewerber und hat immer noch das Potential, die weitaus meisten Wahlkreise zu gewinnen. ${ }^{14}$

In dieser Konstellation kann die stärkste Partei sich über den Weg der Personenwahl fast die Hälfte der Sollsitze sichern, auch wenn ihr nach der Verhältnisrechnung nur etwa ein Drittel der Sollsitze zustehen. Tatsächlich ist das Problem diffiziler, weil ja nicht nur einmal in der Oberzuteilung für die Parteien auf Bundesebene gerechnet wird, sondern zusätzlich pro Partei eine Unterzuteilung auf Länderebene anfällt.

\section{Lösung: 200 Wahlkreise statt 299}

Das strukturelle Problem kann dadurch gelöst werden, dass die Zahl der Wahlkreise von der Hälfte der Sollsitze auf ein

6 Spalte F in Schaubild 1 zeigt die Landesdivisoren. Die Rechnungen für das erste Land Schleswig-Holstein finden sich in den ersten Zeilen der sechs kleineren Tabellen im unteren Teil. Zur Platzersparnis sind nur Quotienten (Spalte N) und Fiktivsitze (Spalte O) ausgedruckt, nicht aber Interimssitze. Ein durchgestrichener Quotient weist darauf hin, dass die Fiktivsitze sich aus den Direktmandaten ergeben.

7 Kopftabelle in Schaubild 2.

8 Pukelsheim, Fußn. 5 [892, 895]; Pukelsheim/Rossi, Fußn. 2 [213]; F. Pukelsheim, Sitzzuteilungsmethoden, Wiesbaden 2015 [75].

9 Mehr dazu in Abschnitt VIII.

10 Gesetz zur Änderung des Wahl- und Abgeordnetenrechts vom 17.03.2008 (BGBl. I, S. 394). Die wesentliche Neuerung im BWG2008 war die Ersetzung des Quotenverfahrens mit Ausgleich nach größten Resten - in Deutschland benannt nach dem Engländer Thomas Hare (1806-1891) und dem Deutschen Horst F. Niemeyer (1931-2007) - durch das Divisorverfahren mit Standardrundung.

11 BVerfGE 95 (1997) 335-407 [Rdnr. 102 f.].

12 Zur verfassungsgerichtlichen Rechtsprechung bis 2010 siehe H. Meyer, Die Zukunft des Bundestagswahlrechts - Zwischen Unverstand, obiter dicta, Interessenkalkül und Verfassungsverstoß, Baden-Baden 2010.

13 BVerfGE 131 (2013) 316-376 [Leitsatz 2.b].

14 Zweitstimmenanteil von $\mathrm{CDU}+\mathrm{CSU}=(12.447 .656+$ 2.869.688)/44.189.959 $=35 \%$. Direktmandatsanteil von CDU + $\mathrm{CSU}=(185+49) / 598=39 \%$. 
Drittel reduziert wird, also von 299 auf 200. Das ist leichter gesagt als getan.

Um die Auswirkungen einer solchen Reduktion in den Blick nehmen zu können, sei eine hypothetische Modellrechnung diskutiert, die so weit wie möglich den Daten der Wahl 2017 folgt. Die Verteilung von 200 Wahlkreisen gemäß den Bevölkerungszahlen in Spalte $C$ von Schaubild 1 stellt sich wie folgt dar: ${ }^{15}$ ständlich ist. Die Vorabkalkulation im BWG2013 ist so strapaziös, dass der Bezug auf die Direktmandate in der Hitze der Rechnung verdunstet.

\section{Direktmandatsorientierte Proporzanpassung: „BWG2013 light"}

Ein Verfahren, das die Untiefen des BWG2013 umschifft, ist die direktmandatsorientierte Proporzanpassung. ${ }^{18}$ Sie ersetzt

\begin{tabular}{|c|c|c|c|c|c|c|c|c|c|c|c|c|c|c|c|c|c|}
\hline & SH & MV & $\mathrm{HH}$ & NI & $\mathrm{HB}$ & $\mathrm{BB}$ & ST & $\mathrm{BE}$ & NW & SN & $\mathrm{HE}$ & TH & $\mathrm{RP}$ & BY & BW & SL & Summe \\
\hline & 11 & 6 & 6 & 30 & 2 & 10 & 9 & 12 & 64 & 16 & 22 & 8 & 15 & 46 & 38 & 4 & 299 \\
\hline Hypothetisch & 7 & 4 & 4 & 20 & 2 & 7 & 6 & 8 & 43 & 11 & 14 & 6 & 10 & 31 & 25 & 2 & 200 \\
\hline
\end{tabular}

Zudem ist in jedem Land eine hypothetische Verteilung der Direktmandate zu bestimmen, um die tatsächlichen Direktmandatsgewinne 2017 auf eine Gesamtheit von nur 200 Wahlkreisen zu projizieren. Die Projektion liefert die hypothetischen Direktmandatszahlen, die in Spalte L von Schaubild 3 eingetragen sind. Die Zweitstimmenergebnisse aus der Wahl 2017 werden unverändert übernommen. Im Folgenden diskutieren wir, was in diesem hypothetischen 200-Wahlkreis-Modell passiert wäre.

\section{Schwäche des BWG2013}

Das BWG2013 hätte im 200-Wahlkreis-Modell zu einem Bundestag mit immer noch 620 Sitzen geführt. Das BWG2013 verpasst die Sollgröße also im hypothetischen Modell genauso wie in der Realität. ${ }^{16}$ Worin ist dieses Versagen begründet?

Das BWG2013 krankt an einer Vorabkalkulation der Bundestagsgröße, die sich in föderalen Nebensächlichkeiten verheddert. Die Rechnung wird dominiert vom kleinsten Landesdivisor, der die Verteilungszahl für die abschließende Oberzuteilung nach unten zieht. So hat in Schaubild 1 Bremen den kleinsten Landesdivisor (64.000), der Bundesdivisor in Schaubild 2 ist sogar noch kleiner (62.300). Die Unterschiede in den Landesdivisoren hängen wesentlich von der Wahlbeteiligung ab. ${ }^{17}$ Wenn in einem Land die Wahlbeteiligung geringer ist als in einem anderen Land, braucht es im ersten Land weniger Stimmen für einen Sitz als im zweiten und folglich wird der erste Wahlschlüssel kleiner sein als der zweite. Das BWG2013 orientiert die Bundestagsgröße also an variierender Wahlbeteiligung, obwohl das Ziel doch ist, eine Einbettung der Direktmandatsgewinne in die Verhältnisrechnung sicher zu stellen. Das eine hat mit dem anderen ziemlich wenig zu tun.

Zudem rackert sich das BWG2013 ab, sogar Parteien ohne Direktmandate mit Mindestsitzzahlen zu beglücken. Die Zahl der Direktmandate bei AfD, FDP, LINKE und GRÜNE ist so gering, dass ein Konflikt mit der Verhältnisrechnung ausgeschlossen ist. Trotzdem kommt das BWG2013 mit eindrucksvollen Mindestsitzzahlen daher (nämlich: 83, 65, 59, 57), die obendrein bei 299 Wahlkreisen dieselben sind wie im 200-Wahlkreis-Modell! Bei der Wahl 2017 ist die CDU letztendlich für die Bundestagsgröße 709 tonangebend, was angesichts der 185 CDU-Direktmandate verständlich ist. Im 200-Wahlkreis-Modell werden die letzten elf Sitze (610-620) nur geschaffen, um die Mindestsitzzahl der AfD unterzubringen, was bei drei AfD-Direktmandaten vollkommen unver- die seitenfüllende Vorabkalkulation des BWG2013 durch einfaches Kopfrechnen: Die Mindestsitzzahl einer Partei ist gleich ihrer bundesweiten Zahl an Direktmandaten plus einem Aufschlag von zehn Prozent. ${ }^{19}$ Danach geht es weiter wie im BWG2013. Die direktmandatsorientierte Proporzanpassung ist ein »BWG2013 light«.

Die direktmandatsorientierte Proporzanpassung für das 200-Wahlkreis-Modell ist in Schaubild 3 dargestellt. Von den hypothetischen 200 Wahlkreisen entfallen 124 auf die CDU. Also werden für die CDU mindestens $124+12=136$ Sitze vorgemerkt (Spalte B). Die hypothetischen zwei AfDWahlkreise führen zur Mindestsitzzahl $2+0=2$. Die FDP bleibt ohne Direktmandat, weshalb sich eine schützende Vormerkung erübrigt: $0+0=0$. Alle Mindestsitzzahlen werden schon bei der Sollgröße von 598 Sitzen befriedigt, also bleibt es dabei. Im 200-Wahlkreis-Modell würde der Bundestag mit der Sollgröße von 598 Sitzen amtieren.

Mehr noch: Bei einer Reduktion auf 200 Wahlkreise hätte die direktmandatsorientierte Proporzanpassung bei allen bisherigen Wahlen die Sollgröße eingehalten. Selbst mit den herkömmlichen 299 Wahlkreisen wäre die Sollgröße meist eingehalten worden, nämlich immer außer 2009 und 2017. ${ }^{20}$ Bei der Wahl 2009 wäre der Bundestag auf 653 Sitze angewachsen, bei der aktuellen Wahl 2017 sogar auf 723 Sitze. Die direktmandatsorientierte Proporzanpassung ist also kein Allheilmittel. Das strukturelle Problem, dass die hälftige Aufteilung in Direkt- und Listenmandate nicht mehr zeitgemäß ist, bleibt vordringlich.

\footnotetext{
15 In $₫ 3(1)$ BWG sollten die Abweichungen der Wahlkreisgrößen nicht auf den Bundesdurchschnitt bezogen werden, sondern auf den Landesdurchschnitt. Zum Beispiel können die zwei Wahlkreise in Bremen nicht gleicher sein als gleich, egal wie der Bundesdurchschnitt ausfällt. S.a. Pukelsheim, Fußn. 8 [73].

16 Das BWG2013 hält überhaupt nie die Sollgröße ein, siehe Fußn. 2.

17 Pukelsheim/Rossi, Fußn. 2 [221].

18 R. Peifer/D. Lübbert/K.-F. Oelbermann/F. Pukelsheim, Direktmandatsorientierte Proporzanpassung - Eine mit der Personenwahl verbundene Verhältniswahl ohne negative Stimmgewichte, DVBl 127 (2012) 725-730.

19 Der Aufschlag sichert die Verfügbarkeit von Listenmandaten für Landeslisten, die im Gegensatz zu anderen Landeslisten nur wenige oder keine Direktmandate aufweisen. Für eine Partei mit nur einer Landesliste entfällt der Aufschlag (CSU). Die Höhe des Aufschlags ist eine politische Entscheidung.
}

20 Pukelsheim/Rossi, Fußn. 2 [222]. 


\begin{tabular}{|l|c|c|c|c|}
\hline \multicolumn{1}{|c|}{ A } & B & C & D & E \\
\hline Partei & mindestens Dir. $+10 \%$ & Zweitstimmen & Quotient & Sitze \\
\hline CDU & $124+12=136$ & 12.447 .656 & 168,4 & 168 \\
\hline SPD & $39+4=43$ & 9.539 .381 & 129,1 & 129 \\
\hline AfD & $2+0=2$ & 5.878 .115 & 79,54 & 80 \\
\hline FDP & $0+0=0$ & 4.999 .449 & 67,7 & 68 \\
\hline LINKE & $3+0=3$ & 4.297 .270 & 58,1 & 58 \\
\hline GRÜNE & $1+0=1$ & 4.158 .400 & 56,3 & 56 \\
\hline CSU & $31+0=31$ & 2.869 .688 & 38,8 & 39 \\
\hline Summe (Bundesdivisor) & $200+16=216$ & 44.189 .959 & $(73.900)$ & 598 \\
\hline
\end{tabular}

\begin{tabular}{|c|c|c|c|c|c|c|c|c|c|c|c|c|c|c|}
\hline $\mathrm{K}$ & $\mathrm{L}$ & $\mathrm{M}$ & $\mathrm{N}$ & $\mathrm{O}$ & $\mathrm{K}$ & $\mathrm{L}$ & $\mathrm{M}$ & $\mathrm{N}$ & $\mathrm{O}$ & $\mathrm{K}$ & $\mathrm{L}$ & M & $\mathrm{N}$ & $\mathrm{O}$ \\
\hline $\begin{array}{l}\text { Landes- } \\
\text { liste }\end{array}$ & Dir. & Zweitst. & Quotient & Sitze & $\begin{array}{l}\text { Landes- } \\
\text { liste }\end{array}$ & Dir. & Zweitst. & Quotient & Sitze & \begin{tabular}{|l|}
$\begin{array}{l}\text { Landes- } \\
\text { liste }\end{array}$ \\
\end{tabular} & Dir. & Zweitst. & Quotient & Sitze \\
\hline & & & & & & & & & & & & & & \\
\hline \multicolumn{5}{|c|}{ Unterzuteilung für die CDU } & \multicolumn{5}{|c|}{ Unterzuteilung für die SPD } & \multicolumn{5}{|c|}{ Unterzuteilung für die AfD } \\
\hline $\mathrm{SH}$ & 6 & 583.135 & 7,7 & 8 & $\mathrm{SH}$ & 1 & 399.505 & 5,4 & 5 & $\mathrm{SH}$ & 0 & 140.362 & 1,9 & 2 \\
\hline MV & 4 & 307.263 & 4,1 & 4 & MV & 0 & 139.689 & 1,9 & 2 & MV & 0 & 172.409 & 2,4 & 2 \\
\hline $\mathrm{HH}$ & 1 & 266.312 & 3,52 & 4 & $\mathrm{HH}$ & 3 & 229.862 & 3,1 & 3 & $\mathrm{HH}$ & 0 & 76.511 & 1,0 & 1 \\
\hline $\mathrm{NI}$ & 11 & 1.623 .481 & 21,4 & 21 & $\mathrm{NI}$ & 9 & 1.275 .172 & 17,1 & 17 & NI & 0 & 422.362 & 5,8 & 6 \\
\hline $\mathrm{HB}$ & 0 & 83.409 & 1,1 & 1 & $\mathrm{HB}$ & 2 & 88.944 & 1,2 & 2 & HB & 0 & 33.244 & 0,46 & 0 \\
\hline $\mathrm{BB}$ & 6 & 397.839 & 5,3 & 6 & $\mathrm{BB}$ & 1 & 261.822 & 3,52 & 4 & BB & 0 & 301.103 & 4,1 & 4 \\
\hline ST & 6 & 377.411 & 5,0 & 6 & ST & 0 & 188.980 & 2,54 & 3 & ST & 0 & 244.401 & 3,3 & 3 \\
\hline $\mathrm{BE}$ & 3 & 424.321 & 5,6 & 6 & $\mathrm{BE}$ & 2 & 334.253 & 4,49 & 4 & $\mathrm{BE}$ & 0 & 225.170 & 3,1 & 3 \\
\hline $\mathrm{NW}$ & 26 & 3.214 .013 & 42,46 & 42 & NW & 17 & 2.557 .876 & 34,4 & 34 & NW & 0 & 928.425 & 12,7 & 13 \\
\hline SN & 8 & 665.751 & 8,8 & 9 & $\mathrm{SN}$ & 0 & 261.105 & 3,51 & 4 & SN & 2 & 669.940 & 9,2 & 9 \\
\hline $\mathrm{HE}$ & 11 & 1.033 .200 & 13,6 & 14 & $\mathrm{HE}$ & 3 & 788.427 & 10,6 & 11 & HE & 0 & 398.712 & 5,46 & 5 \\
\hline $\mathrm{TH}$ & 6 & 372.258 & 4,9 & 6 & $\mathrm{TH}$ & 0 & 171.032 & 2,3 & 2 & TH & 0 & 294.069 & 4,0 & 4 \\
\hline $\mathrm{RP}$ & 9 & 848.003 & 11,2 & 11 & $\mathrm{RP}$ & 1 & 570.518 & 7,7 & 8 & RP & 0 & 265.688 & 3,6 & 4 \\
\hline $\mathrm{BY}$ & - & - & - & - & $\mathrm{BY}$ & 0 & 1.130 .931 & 15,2 & 15 & BY & 0 & 916.300 & 12,6 & 13 \\
\hline BW & 25 & 2.061 .687 & 27,2 & 27 & $\mathrm{BW}$ & 0 & 982.370 & 13,2 & 13 & BW & 0 & 730.499 & 10,0 & 10 \\
\hline SL & 2 & 189.573 & 2,504 & 3 & SL & 0 & 158.895 & 2,1 & 2 & SL & 0 & 58.920 & 0,8 & 1 \\
\hline Sa. (Div.) & 124 & 12.447 .656 & $(75.700)$ & 168 & Sa. (Div.) & 39 & 9.539 .381 & $(74.400)$ & 129 & Sa. (Div.) & 2 & 5.878 .115 & $(73.000)$ & 80 \\
\hline & & & & & & & & & & & & & & \\
\hline \multicolumn{5}{|c|}{ Unterzuteilung für die FDP } & \multicolumn{5}{|c|}{ Unterzuteilung für die LINKE } & \multicolumn{5}{|c|}{ Unterzuteilung für die GRÜNEN } \\
\hline $\mathrm{SH}$ & 0 & 216.844 & 3,1 & 3 & $\mathrm{SH}$ & 0 & 124.678 & 1,7 & 2 & $\mathrm{SH}$ & 0 & 205.471 & 2,7 & 3 \\
\hline MV & 0 & 57.895 & 0,8 & 1 & MV & 0 & 165.368 & 2,2 & 2 & MV & 0 & 39.514 & 0,53 & 1 \\
\hline $\mathrm{HH}$ & 0 & 105.610 & 1,49 & 1 & $\mathrm{HH}$ & 0 & 119.076 & 1,6 & 2 & $\mathrm{HH}$ & 0 & 136.371 & 1,8 & 2 \\
\hline $\mathrm{NI}$ & 0 & 431.405 & 6,1 & 6 & $\mathrm{NI}$ & 0 & 322.979 & 4,3 & 4 & NI & 0 & 404.825 & 5,4 & 5 \\
\hline $\mathrm{HB}$ & 0 & 31.056 & 0,4 & 0 & $\mathrm{HB}$ & 0 & 44.629 & 0,6 & 1 & $\mathrm{HB}$ & 0 & 36.733 & 0,49 & 0 \\
\hline $\mathrm{BB}$ & 0 & 105.485 & 1,49 & 1 & BB & 0 & 255.721 & 3,4 & 3 & BB & 0 & 74.971 & 1,0 & 1 \\
\hline ST & 0 & 96.555 & 1,4 & 1 & ST & 0 & 220.858 & 2,9 & 3 & ST & 0 & 46.243 & 0,6 & 1 \\
\hline $\mathrm{BE}$ & 0 & 167.046 & 2,4 & 2 & $\mathrm{BE}$ & 2 & 351.170 & 4,7 & 5 & $\mathrm{BE}$ & 1 & 234.947 & 3,1 & 3 \\
\hline $\mathrm{NW}$ & 0 & 1.293 .052 & 18,2 & 18 & NW & 0 & 736.904 & 9,8 & 10 & NW & 0 & 744.970 & 9,9 & 10 \\
\hline SN & 0 & 203.662 & 2,9 & 3 & SN & 1 & 398.627 & 5,3 & 5 & SN & 0 & 113.608 & 1,51 & 2 \\
\hline $\mathrm{HE}$ & 0 & 386.742 & 5,4 & 5 & $\mathrm{HE}$ & 0 & 271.158 & 3,6 & 4 & $\mathrm{HE}$ & 0 & 323.736 & 4,3 & 4 \\
\hline TH & 0 & 101.129 & 1,4 & 1 & $\mathrm{TH}$ & 0 & 218.212 & 2,9 & 3 & TH & 0 & 53.340 & 0,7 & 1 \\
\hline $\mathrm{RP}$ & 0 & 245.235 & 3,45 & 3 & $\mathrm{RP}$ & 0 & 160.912 & 2,1 & 2 & RP & 0 & 179.233 & 2,4 & 2 \\
\hline BY & 0 & 751.248 & 10,6 & 11 & BY & 0 & 450.803 & 6,0 & 6 & BY & 0 & 722.116 & 9,6 & 10 \\
\hline $\mathrm{BW}$ & 0 & 762.008 & 10,7 & 11 & $\mathrm{BW}$ & 0 & 380.727 & 5,1 & 5 & BW & 0 & 807.205 & 10,8 & 11 \\
\hline SL & 0 & 44.477 & 0,6 & 1 & SL & 0 & 75.448 & 1,0 & 1 & SL & 0 & 35.117 & 0,47 & 0 \\
\hline Sa. (Div.) & 0 & 4.999 .449 & $(71.000)$ & 68 & Sa. (Div.) & 3 & 4.297 .270 & $(75.000)$ & 58 & \begin{tabular}{|l|} 
Sa. (Div.) \\
\end{tabular} & 1 & 4.158 .400 & $(75.000)$ & 56 \\
\hline
\end{tabular}

\section{Schaubild 3: Modellrechnung mit 200 Wahlkreisen und direktmandatsorientierter Proporzanpassung}

Die Modellrechnung unterstellt 200 Wahlkreise. In jedem Land sind die Direktmandatszahlen (Spalte L) hypothetisch berechnet gemäß dem Verhältnis der tatsächlichen Direktmandatsgewinne von 2017; die Zweitstimmenzahlen sind unverändert übernommen. Die direktmandatsorientierte Proporzanpassung gelangt zur Mindestsitzzahl einer Partei, indem die Zahl ihrer bundesweiten Direktmandate um zehn Prozent erhöht wird (Spalte B). Mit der Oberzuteilung von 598 Sitzen werden alle Mindestsitzzahlen befriedigt (Spalte E): Aufje 73.900 Zweitstimmen entfällt rund ein Sitz. Die Unterzuteilungen an die Landeslisten beruhen auf demselben Verfahren, das im BWG2013 festgelegt ist. 


\section{Föderale Unproportionalitäten}

Für die verfassungsrechtliche Beurteilung von Sitzzuteilungsverfahren ist der zu betreibende Rechenaufwand natürlich zweitrangig. Ausschlaggebend ist, ob die produzierten Sitzzuteilungen in Einklang stehen mit den Wahlgrundsätzen.

Für die Oberzuteilung, also die Vergabe der Gesamtsitze an die Parteien gemäß bundesweiten Zweitstimmenergebnissen, ist Verfassungskonformität sowohl für das BWG2013 wie auch für die direktmandatsbedingte Proporzanpassung uneingeschränkt zu bejahen. Beide Anwendungen beruhen auf dem Divisorverfahren mit Standardrundung, das mit den Wahlgrundsätzen, insbesondere mit dem Grundsatz der gleichen Wahl, hervorragend harmoniert. ${ }^{21}$ Dies gilt auch bei den Unterzuteilungen für diejenigen Parteien, bei denen die Bedingtheit durch Direktmandate inaktiv bleibt (AfD, FDP, LINKE, GRÜNE).

Nur bei den Unterzuteilungen für CDU und SPD treten föderale Unproportionalitäten auf, deren verfassungsrechtliche Verträglichkeit prüfungswürdig ist. ${ }^{22} \mathrm{Da}$ das Bundesverfassungsgericht traditionsgemäß auf Mandatserheblichkeit abstellt, drücken wir die abstrakte Idee von »Unproportionalität« konkret durch die Zahl der Sitztransfers aus, deren es bedarf, um von einer gegebenen Sitzzuteilung überzugehen zu einer proportionalen Sitzzuteilung. Als Beispiel diene die Unterzuteilung für die SPD in Schaubild 3. Diese weist der SPD-Landesliste in Bremen zwei Sitze zu. Eine Zuteilung aller 129 SPD-Sitze mit dem Divisorverfahren mit Standardrundung (also ohne Direktmandatsbedingungen) hätte Bremen einen Sitz gegeben statt zwei und Berlin fünf statt vier. Die Unproportionalität der SPD-Unterzuteilung beträgt Eins, weil auf dem Weg zur proportionalen Sitzzuteilung ein Sitz transferiert werden muss.

Die Unproportionalitäten, die im amtierenden 19. Bundestag Realität sind, belaufen sich auf sechzehn Transfers bei den 200 CDU-Sitzen und auf zwei Transfers bei den 153 SPDSitzen.

Im hypothetischen 200-Wahlkreis-Modell wären es bei Anwendung des BWG2013 zwei Transfers bei den dann 175 CDU-Sitzen und ein Transfer bei den 134 SPD-Sitzen.

Bei der Modellrechnung mit der direktmandatsorientierten Proporzanpassung würde die Unproportionalität bei den 168 CDU-Unterzuteilung drei Transfers ausmachen und bei den 129 SPD-Sitzen den eingangs erläuterten einen Transfer.

Die föderalen Unproportionalitäten, die Realität sind, haben bislang zu keinerlei Beanstandungen Anlass gegeben. Also dürften wohl auch die geringeren Unproportionalitäten nach einer Wahlkreisreduktion fraglos tolerierbar sein, sowohl wenn das derzeitige BWG2013 fortdauert als auch wenn zukünftig zum »BWG2013 light« gewechselt wird.

\section{Ausblick}

Im Ergebnis halten wir fest, dass das Wahlsystem für den Bundestag an die gewandelten gesellschaftlichen Verhältnisse angepasst werden sollte. Wenn das Gesetz eine Regelgröße von 598 Bundestagssitzen vorgibt, dann sollten die Bürgerinnen und Bürger das Gesetz auch ernst nehmen dürfen.
Die derzeitige Übergröße von 709 Sitzen geht nicht mit einer übergroßen Ernsthaftigkeit an demokratischer Repräsentation einher. Eher werden Zweifel an der Kompetenz des Gesetzgebers geweckt. Immerhin steht für das Europäische Parlament, das fast eine halbe Milliarde Unionsbürger repräsentiert statt nur achtzig Millionen Deutsche, nach dem Ausscheiden des Vereinigten Königreichs eine Verkleinerung von 751 auf 700 Sitze im Raum. ${ }^{23}$ Ein Bundestag mit 709 Sitzen erscheint dem gegenüber maßlos.

Die in diesem Aufsatz favorisierte Lösung des Strukturproblems ist die Verringerung der Zahl der Wahlkreise von 299 auf 200. Unsere Ausführungen zeigen, dass damit die vom Gesetz vorgegebene Sollgröße immer eingehalten wird. ${ }^{24}$ Die verfahrensimmanente Analyse ist aber eher nur eine notwendige und sicherlich keine hinreichende Bedingung für die politische Machbarkeit. Aus politischer Sicht ist die Reduzierung der Zahl der Wahlkreise ein Kraftakt. Für einen vergleichbaren Kraftakt in der 13. Wahlperiode, nämlich der Herabsetzung der Bundestagsgröße von 656 auf 598 Sitze, wurde eine Reformkommission eingesetzt, um auch denkbare Alternativen eingehend zu prüfen und zu würdigen.

Denkbar ist, umfassender in das geltende Wahlrecht einzugreifen. Man könnte das Zwei-Stimmen-System durch eine andere Stimmgebung ersetzen und dementsprechend die Sitzzuteilungsmethode neu festlegen. ${ }^{25}$ Jeder Wechsel auf eine andere Stimmgebung bedarf allerdings eine Umerziehung des Wahlvolks, die akribisch vorbereitet werden muss. Bei der hier vorgeschlagenen Lösung, nur ein Drittel der Sitze über Wahlkreise zu besetzen und zur direktmandatsorientierten Proporzanpassung überzugehen, ändert sich für die Wähler und Wählerinnen nichts. Sie können ihr Wahlverhalten unverändert beibehalten.

Allerdings würden die "Anpassungskosten « für unseren Vorschlag alleinig den Abgeordneten aufgebürdet. Großräumigere Wahlkreise bedeuten Mehrarbeit nicht nur für diejenigen Abgeordnete, die einen Wahlkreis direkt vertreten, sondern auch für die anderen, die zwar über Listen in den Bundestag einziehen, aber ihre politische Basisarbeit an den gegebenen Wahlkreisen orientieren. Andererseits sind Strukturanpassungen nicht nur in Deutschland vonnöten, sondern anderswo auch. Das neue italienische Wahlgesetz sieht von vorne herein vor, dass nicht die Hälfte, sondern nur ein Drittel der Sitze direkt in Wahlkreisen vergeben werden. ${ }^{26}$

21 Siehe Fußn. 8.

22 Pukelsheim/Rossi, Fußn. 2 [219].

23 D.M. Hübner/P. Silva Pereira, Draft Report on the Composition of the European Parliament (2017/2054[INL]), Committee on Constitutional Affairs of the European Parliament, PE 608.038v01-00, 7 September 2017.

24 Der Neuzuschnitt der Wahlkreise kann durch Computerprogramme unterstützt werden, siehe zum Beispiel S. Goderbauer, Mathematische Optimierung der Wahlkreiseinteilung für die Deutsche Bundestagswahl - Modelle und Algorithmen für eine bessere Beachtung der gesetzlichen Vorgaben, Wiesbaden 2016.

25 Siehe zum Beispiel J. Behnke, Ein sparsames länderproporzoptimierendes parteienproporzgewährendes automatisches Mandatszuteilungsverfahren mit Ausgleich ohne negatives Stimmgewicht. ZParl 43 (2012) 675-693, V. Schröder: Parteienproporz, Länderproporz und Bundestagsgröße im neuen Bundestagswahlrecht. ZParl 45 (2014) 838-858, P. Weinmann: Zweierwahlkreise als Mittel gegen übergroße Bundestage? ZPol 24 (2014) 449-489.

26 L. Borsi/S. Bonanni/M. Frati, Riforma Elettorale, Note sull'A.S. n. 2941 (schede di lettura; testo a fronte), Servizio Studi del Senato, Roma Ottobre 2017. 


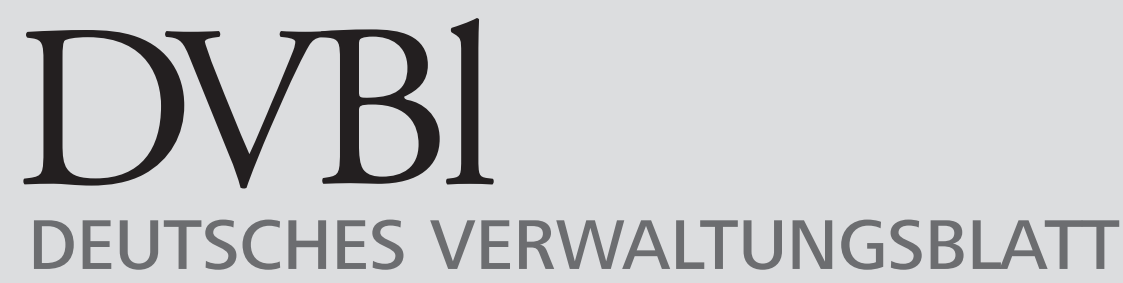

\section{INHALT $3 \cdot 2018$}

DVBI aktuell

Vorschau/Impressum

\section{Aufsätze}

„Die Kreisumlage darf nicht zu einer nachhaltigen Verkürzung der gemeindeeigenen Finanzbasis führen"

Geschäftsführendes Präsidialmitglied des Deutschen Landkreistages Prof. Dr. Hans-Günter Henneke, Berlin/Universität Osnabrück

133

A-limine-Entscheidungen in Wahlprüfungsangelegenheiten - Eine Analyse der Rechtsprechung des Zweiten Senats des Bundesverfassungsgerichts zur Gültigkeit der Bundestagswahl 2013

Prof. Dr. Wolfgang Schreiber, Bonn

598 Sitze im Bundestag statt 709? 200

Wahlkreise statt 299!

Prof. Dr. Friedrich Pukelsheim, Augsburg

Grenzüberschreitende Umweltprüfungen Änderungen durch das Gesetz zur Modernisierung des Rechts der Umweltverträglichkeitsprüfung Regierungsdirektor Dr. René Grandjot, Berlin

Divergenzen beim Grundrechtsschutz in Karlsruhe und Straßburg

Prof. Dr. Jürgen Schwabe, Hamburg

\section{Buchbesprechungen}

Frenzel (Hrsg.): Was bleibt

Prof. Dr. Dr. Jörg Berkemann, Hamburg/Berlin

Faber: Föderalismus und Binnenföderalismus im Wahlrecht zu den deutschen Volksvertretungen und zum Europäischen Parlament

Prof. (em.) Dr. iur. Herbert Bethge, Passau

Schneider/Schwarz (Hrsg.): Parlamentarische Opposition zwischen Effektivität und Egalität Ministerialrat Dr. Michael Fuchs, M. A., Magister rer. publ., Berlin

von Arnim: Die Hebel der Macht und wer sie bedient. Parteienherrschaft statt Volkssouveränität Prof. Dr. jur. Klaus Schönenbroicher, Düsseldorf
III Lauer: Das Recht des Beamten zum Streik

VII Prof. (em.) Dr. Dr. h.c. Ulrich Battis, Berlin

Schoch/Schneider/Bier (Hrsg.):

Verwaltungsgerichtsordnung

Prof. Dr. Friedhelm Hufen, Mainz

Kopp/Ramsauer: Verwaltungsverfahrensgesetz Vizepräsident des VG Köln Andreas Becker, BonnBad Godesberg/Köln

\section{Rechtsprechung}

\section{Bundesverfassungsgericht}

BVerfG, 4. K. des 1. Sen., Beschl. v. 20.12.2017 - 1 BvR 2754/17 -

Einstweilige Anordnung gegen Bestellung eines Sonderprüfers im VW-Dieselskandal abgewiesen

BVerfG, 2. K. des 1. Sen., Beschl. v. 24.10.2017 - 1 BvR 1026/13 - u.a.

Erfolglose Vb gegen die Planfeststellung Flughafen Schönefeld

\section{Landesverfassungsgericht}

[LS] VerfGH NRW, Urt. v. 21.11.2017-15/16-

2,5\%-Sperrklausel in NRW ist verfassungsgemäß

\section{Bundesverwaltungsgericht}

BVerwG, Urt. v. 13.09.2017 - 10 C 7.16 -

Freier Zugang zum Meeresstrand

BVerwG, Urt. v. 29.06.2017 - 3 A $1.16-$

Anfechtung eines eisenbahnrechtlichen

Planfeststellungsbeschlusses (Dresdner Bahn)

\section{Oberverwaltungsgerichte/ Verwaltungsgerichtshöfe}

Hess VGH, Urt. v. 26.10.2017 - 9 C 873/15.T - n.r. Keine Klagebefugnis Lärmbetroffener in Bezug auf einen Lärmaktionsplan

OVG NRW, Urt. v. 06.10.2017-11 A 1159/15-

Rückbau einer Gehwegüberfahrt ist keine Sondernutzung

Nds OVG, Beschl. v. 07.12.2017 - 12 ME 163/17 Fehlende Antragskonkurrenz zwischen Vorbescheid und Genehmigungsbescheid 